\title{
Phenotypic plasticity in sperm production rate: there's more to it than testis size
}

\author{
Lukas Schärer · Dita B. Vizoso
}

Received: 8 December 2005/ Accepted: 20 July 2006/ Published online: 31 August 2006

(C) Springer Science+Business Media B.V. 2006

\begin{abstract}
Evolutionary theory predicts that males should produce more sperm when sperm competition is high. Because sperm production rate is difficult to measure in most organisms, comparative and experimental studies have typically used testis size instead, while assuming a good correspondence between testis size and sperm production rate. Here we evaluate this common assumption using the marine flatworm Macrostomum lignano, in which we can estimate sperm production rate because the accumulation of produced sperm can be observed in vivo. In earlier studies we have shown that testis size is phenotypically plastic in M. lignano: worms can be induced to make larger testes by raising them in groups instead of pairs, and these larger testes have a higher cell proliferation activity (i.e. they are more energetically costly). Here we demonstrate that worms with such experimentally enlarged testes have a higher sperm production rate. Moreover, although testis size and sperm production rate were related linearly, worms with experimentally enlarged testes had a higher sperm production rate per unit testis size (i.e. a higher spermatogenic efficiency). We thus show that phenotypically plastic adjustment of sperm production rate includes a component that is independent of testis size. We discuss possible reasons for this novel finding, and suggest that the relationship between testis size and sperm production needs to be evaluated in other species as well.
\end{abstract}

Keywords Cell cycle $\cdot$ Male allocation $\cdot$ Phenotypic plasticity $\cdot$ Platyhelminthes $\cdot$ Sperm competition $\cdot$ Testis size

\section{Introduction}

Current evolutionary theory predicts that the amount of sperm a male produces depends on the level and the type of sperm competition it experiences (Parker 1998).

L. Schärer $(\bowtie) \cdot$ D. B. Vizoso

Division of Ultrastructural Research and Evolutionary Biology, Institute of Zoology,

University of Innsbruck, Technikerstrasse 25, 6020 Innsbruck, Austria

e-mail: lukas.scharer@uibk.ac.at 
Comparative and experimental studies aimed at testing this prediction have generally used testis size as a measure of sperm production rate (see Schärer et al. 2004b for a list of parameters that have been used to estimate male allocation to sperm production). The choice of this parameter is largely guided by the relative ease with which it can be determined, but it has rarely been tested how testis size is related to sperm production rate; a linear relationship is usually just assumed. In fact, many studies use testis size and sperm production interchangeably without testing this assumption (e.g. Pitnick et al. 2001; Byrne et al. 2002; Newlon et al. 2003; Malo et al. 2005; Minder et al. 2005), whereas other studies explicitly state that they make this assumption (e.g. Hellriegel and Blanckenhorn 2002).

There are two main problems with using testis size as a measure of sperm production rate. First, testis size is a static measure, and therefore does not necessarily say much about the amount of resources that flow through it. We have previously discussed this issue (Schärer et al. 2004b) and we will therefore not further address it here. Second, testis size and sperm production rate may, at least to some degree, vary independently of each other. For example, in mammals the testis not only contains spermatogenic tissues, but also the capsule (or tunica albuginea), which encloses the other testicular tissues, and the Leydig cells, which are involved in the production of male hormones. So, depending on how non-spermatogenic and spermatogenic tissues scale with increasing testis size, the relationship between testis size and sperm production rate will vary. As a consequence, the spermatogenic efficiency of the testis (a measure of the number of sperm produced per unit of testicular tissue) can vary considerably. For instance, although dominant male capybaras have larger testes, their testis size is more closely related to the size of the non-spermatogenic, presumably hormone-producing, tissue than to the amount of spermatogenic tissue in their testis (Moreira et al. 1997) (the high hormone levels are required for the androgen-dependent scent glands). Thus in capybaras spermatogenic efficiency in large testes is relatively low.

Spermatogenic efficiency may thus be an important parameter for sperm production rate. A comparative study in primates showed considerable variation in spermatogenic efficiency between species (Wistuba et al. 2003), and similar changes can also occur within a species, for example if rats are treated with certain chemicals (Cooke et al. 1992). Moreover, a selection experiment for increased testis weight in pigs yielded not only a higher testis weight in the selected line compared to the control line, but also a higher spermatogenic efficiency (Johnson et al. 1994; Rathje et al. 1995), suggesting that this trait can respond to selection. And finally, another study showed different spermatogenic efficiencies in two species of rodents that differ in the level of sperm competition (Peirce and Breed 2001). All these studies suggest that the relationship between testis size and sperm production rate can vary, both within and between species. Spermatogenic efficiency could therefore also vary as a (phenotypically plastic or evolutionary) response to sperm competition. If so, changes in sperm production rate could be, to some degree, independent of testis size, and it would be preferable to measure sperm production rate directly.

However, there are a number of problems involved in the estimation of sperm production rate. A number of studies have used static measures, such as the number of sperm present in the testis (or associated structures) at a given time, as an estimate of sperm production (e.g. Gage et al. 1995; Tuttle et al. 1996; Evans and Magurran 1999; Pitnick et al. 2001). However, in addition to the problems associated with static measures (Schärer et al. 2004b), these measures can be influenced by the 
mating history of an individual or its holding conditions (Bozynski and Liley 2003; Aspbury and Gabor 2004a, b). Other studies have attempted to measure sperm production rate by sequentially mating males to many females and estimating the total amount of sperm transferred (e.g. Pitnick and Markow 1994a, b). However, this approach can be confounded by mate choice, with females accepting more sperm from some males than from others (i.e. Pitnick and Markow 1994a). Mating rate has also been used as a proxy for sperm production rate, but it has been shown that males can run out of sperm in subsequent matings (Nakatsuru and Kramer 1982; Pitnick and Markow 1994b), and that they tailor the number of sperm released in subsequent matings to different conditions (Pilastro et al. 2002; Wedell et al. 2002). So all of these approaches may be unsuitable measures for sperm production rate.

To our knowledge, accurate data on sperm production rates have so far almost exclusively been collected in studies that investigate fertility in the context of contraception, toxicology and animal breeding, and they are generally limited to birds and mammals (e.g. de Reviers and Williams 1984; Cooke et al. 1996; Gupta et al. 1999, reviewed in Amann (1970), but see LaMunyon and Ward, 1998; Tuttle and Pruett-Jones, 2004 for studies with an evolutionary background). The estimation of sperm production rate is generally based on the number of specific spermatogenetic stages in homogenates of testicular tissue (usually by counting the number of homogenization-resistant spermatid nuclei), combined with accurate knowledge of the duration of spermatogenesis (usually determined using ${ }^{3} \mathrm{H}$-thymidine-, or more recently halogenated pyrimidine-, DNA-labelling, for details see Amann 1970). However, even in the well studied higher vertebrates it is "... clear that testicular weight accounts for no more than about $80 \%$ of the variation in daily sperm production" (Amann 1970) (see Møller 1989 for a similar result). To investigate if the remaining variation is just due to measurement error or due to factors that have been shaped by sperm competition is surely an interesting research avenue.

Here we experimentally evaluate this possibility, by testing the relationship between testis size and sperm production rate in the free-living marine flatworm Macrostomum lignano (Ladurner et al. 2005). Several traits allow such an approach in our model organism. First, we have previously shown that worms can be experimentally induced to produce larger testes by raising them in groups instead of pairs (Schärer and Ladurner 2003; Schärer et al. 2005; Brauer et al. in press), and we have shown that this increase in testis size coincides with a higher cell proliferation activity in the testis (Schärer et al. 2004b). This means that we can experimentally manipulate male reproductive allocation in an otherwise homogenous population. Second, because $M$. lignano is transparent, it is possible to accurately, repeatedly and non-invasively measure the size of internal organs in living worms (Schärer and Ladurner 2003). This allows us to determine not only the testis size of experimental worms, but also the size of their seminal vesicle, which contains the sperm that are ready to be transferred to mating partners. The increase in the size of the seminal vesicle over time can thus serve as an estimate of sperm production rate (Fig. 1). Third, based on ultrastructural observations of the testis, we know that the testis is almost entirely composed of spermatogenic tissue. The only cells that do not directly contribute to spermatogenesis are the hull cells, which delimit the testis from the remaining body, and which make up less than $5 \%$ of the testis volume (L. Schärer, pers. obs.).

In the experiment we report here we raised worms in different group sizes and thereby induced variation in male allocation and testis size. We then isolated indi- 

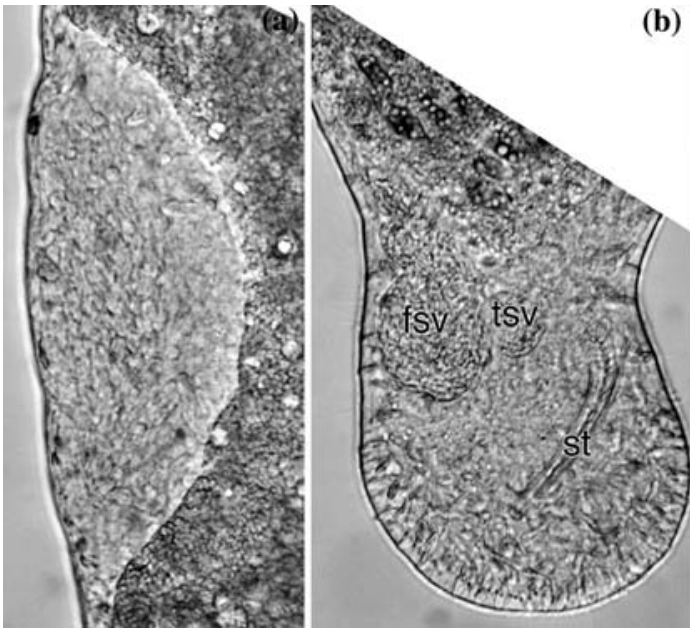

(b)

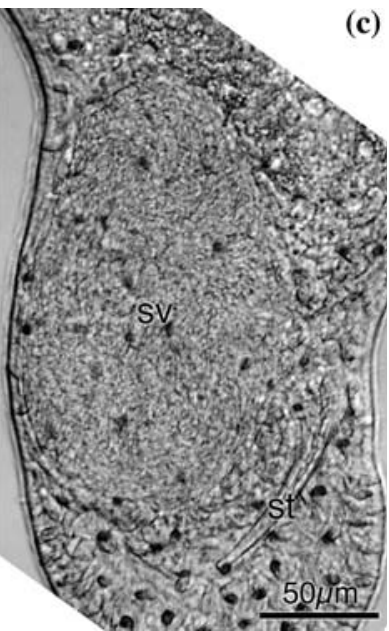

Fig. 1 Digital micrographs of one Macrostomum lignano from the experiment on sperm production rate, which had been raised in a group of eight worms: (a) shows the left testis on Day 0 (light area), (b) shows the seminal vesicle on Day 0 (which consists in the small false seminal vesicle, fsv and the true seminal vesicle, tsv), and (c) shows the drastically larger seminal vesicle (sv) on Day 4 (i.e. after 4 days of isolation) (the false and true seminal vesicles can no longer be distinguished). Note also the copulatory stylet (st). All micrographs were taken at the same scale

viduals from their mating partners and measured the increase in size of the seminal vesicle over time as a measure of sperm production rate. We expected to find that worms with experimentally enlarged testes have a higher sperm production rate.

\section{Methods}

Study animal

Macrostomum lignano (Macrostomorpha, Platyhelminthes) is a free-living flatworm of the interstitial sand fauna of the Northern Adriatic Sea (Ladurner et al. 2000, 2005). It is a simultaneous hermaphrodite with reciprocal copulation (Schärer et al. 2004a), obligately outcrossing (Schärer and Ladurner 2003), reaches $1.5 \mathrm{~mm}$ in length when fully grown, and has a generation time of only 18 days. The prominent paired testes (Fig. 1a) are located anterior to the smaller paired ovaries. Single vasa deferentia emanate from each testis, which join each other shortly before entering the false seminal vesicle (which is essentially a distended common vas deferens, Fig. 1b). The false seminal vesicle in turn is connected to the muscular true seminal vesicle (which pumps the sperm into the copulatory stylet during copulation, Fig. 1b). The bulk of the sperm that are ready to be transferred to mating partners is stored in the very flexible false seminal vesicle (Fig. 1b, c). Mass cultures of Macrostomum lignano are maintained in $\mathrm{f} / 2$, which is an artificial seawater medium (Andersen et al. 2005), and fed with the diatom, Nitzschia curvilineata (Tyler 1981; Rieger et al. 1988). 
Experiment on sperm production rate

On day 1 we collected about 600 adult worms from the mass cultures and allowed them to lay eggs in a Petri dish for $24 \mathrm{~h}$. On day 2 we removed the adults, on day 8 we collected the hatched worms, passed them to a clean Petri dish, and on day 9 we distributed them randomly to pairs or octets. This treatment successfully manipulates the number of sperm competitors (Schärer and Ladurner 2003). We achieved random distribution by pipetting individual worms to their assigned wells in 6-well tissue culture plates according to a permutation of all the wells and treatments, effectively avoiding sequence and position effects. We replicated each treatment group 30 times $(n=60)$, for a total of ten 6 -well tissue culture plates. We fed the worms ad libitum and transferred them to fresh tissue culture plates on days 16, 22, and 29. On day 31 (first measurement, further called "Day 0") we randomly chose one worm per replicate to determine the relationship between testis size and sperm production rate.

Measurement of testis size, seminal vesicle size, and sperm production rate

We morphometrically determined the area of the body, the testis (sum of both testes), and the seminal vesicle (sum of the false and true seminal vesicle) as previously reported (Schärer and Ladurner 2003; Schärer et al. 2005, see also Fig. 1). We have previously shown that these measurements are highly repeatable (Schärer and Ladurner 2003). The person who took the digital micrographs and performed the morphometry was blind regarding the treatment groups of the worms. After measurement, we placed worms individually in wells of 24-well tissue culture plates, and measured them again 2 and 4 days later (called "Day 2" and "Day 4" respectively). During this time the sperm produced in the testes flowed into, and accumulated in, the seminal vesicle (Fig. 1c). Measuring the increase in seminal vesicle size thus allowed us to estimate the sperm production rate of each individual worm.

\section{Relationship between sperm number and seminal vesicle size}

For the increase in seminal vesicle area to be a useful measure of sperm production rate, we need to understand the relationship between the number of sperm contained in the seminal vesicle and the area of the seminal vesicle. To determine this relationship we measured the area of the seminal vesicle of worms with a large range of different seminal vesicle sizes, which was produced by isolating worms for different amounts of time. We then amputated the tail plate with a razor blade, placed the amputated tail plate on a microscope slide in $17 \mu \mathrm{l}$ of $\mathrm{f} / 2$, ruptured the seminal vesicle with fine tungsten needles to release the sperm, covered the drop with a cover slip $(18 \times 18 \mathrm{~mm})$ and counted all sperm under phase contrast illumination.

\section{Statistical analysis}

We discarded three pair treatment replicates because worms were lost during the transfers. Moreover, several worms were excluded because they lacked a functional copulatory stylet $(n=2)$, had a hypertrophic testis $(n=2)$, or exhibited other malformations $(n=4)$. The analysis is thus based on 49 worms (pairs, $n=24$ and octets, $n=25$ ). 
To describe the relationship between the number of sperm contained in the seminal vesicle and its area we used a linear regression. To test the effects of the group size treatment we first checked if the two treatment levels yielded worms of comparable body size on the first measurement day using a $t$-test. We then tested the treatment effect on testis size by comparing worms from pairs and octets with an ANCOVA (with body size as the covariate). The treatment effect on the repeated measures of seminal vesicle size was tested with a univariate repeated measures ANOVA. Finally, we investigated if the treatment group of a worm and its testis size independently influenced the size increase of the seminal vesicle from "Day 0" to "Day 4", by calculating an ANCOVA with group size as the factor and testis size as the covariate. Data are given as means $\pm 1 \mathrm{SE}$ and we visually checked that data fulfilled the assumptions of parametric test statistics. A non-significant interaction term was always removed from the ANCOVA models. Data were analysed with JMP 3.2.2 (Sall and Lehman 1996).

\section{Results}

Relationship between sperm number and seminal vesicle size

The number of sperm in the seminal vesicle was a strong predictor of seminal vesicle size (linear regression, $n=38, r^{2}=0.77, b=9.36, t=10.9, P<0.001$, Fig. 2 ), and the significant intercept suggests that also empty seminal vesicles have a certain size ( $a=2930, t=6.11, P<0.001$, Fig. 2). We can thus use the increase in seminal vesicle size as an estimate of sperm production rate.

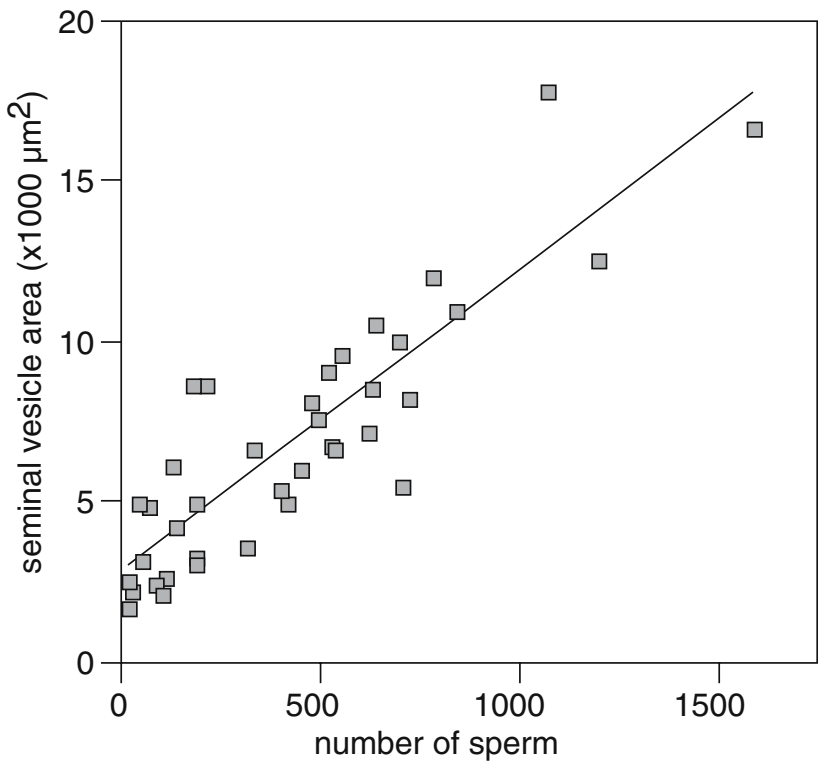

Fig. 2 Seminal vesicle size as a function of the number of sperm in the seminal vesicle (see Results for statistics) 


\section{Experiment on sperm production rate}

Worms in pairs and octets were very similar in body size (body area: pairs, $393 \pm 16 \times 10^{3} \mu \mathrm{m}^{2}$, octets, $406 \pm 16 \times 10^{3} \mu \mathrm{m}^{2}$, $t$-test, $t=0.54$, d.f. $\left.=47, P=0.59\right)$, and, in accordance to our previous findings, testis size was significantly higher in
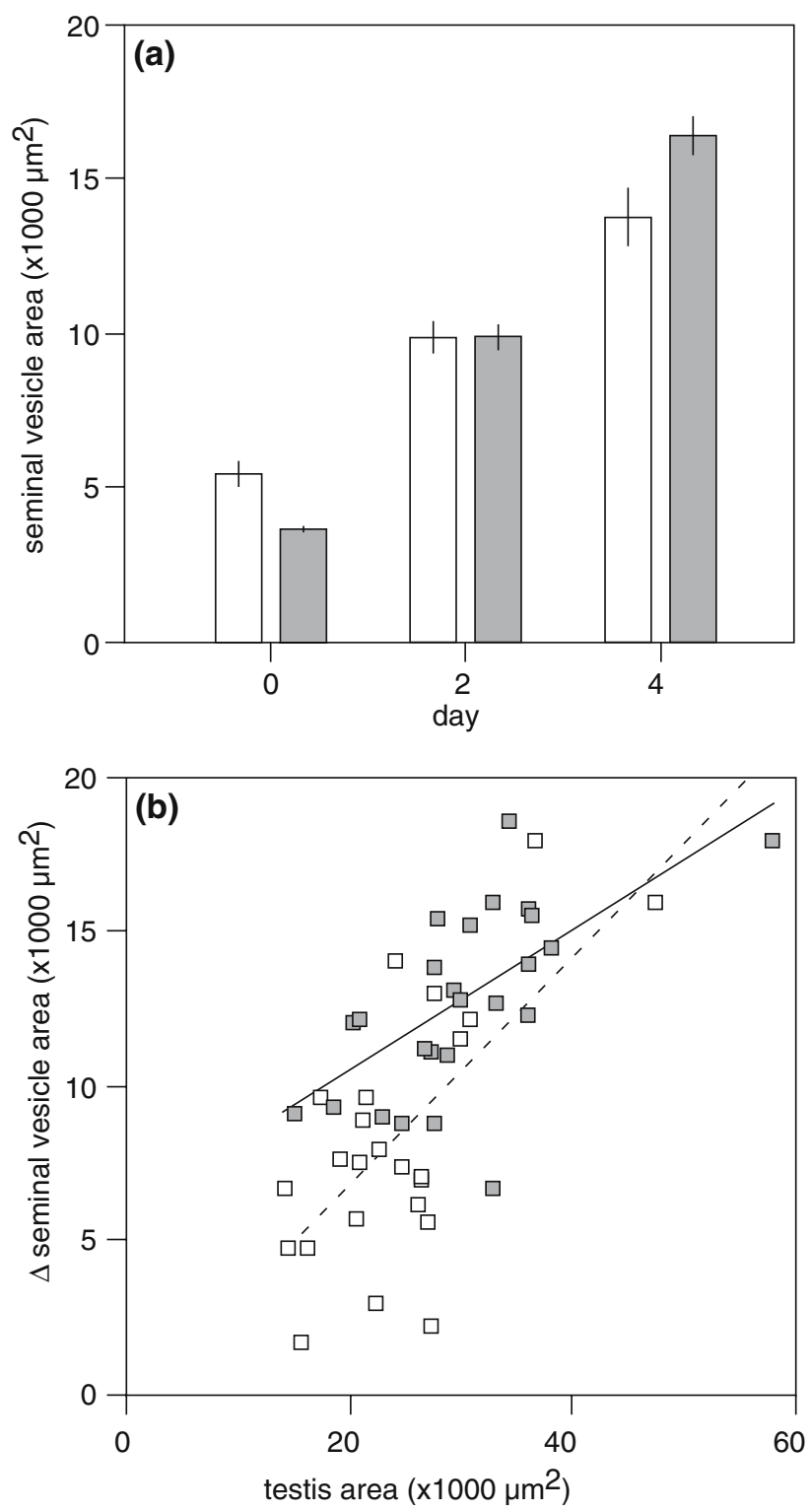

Fig. 3 (a) Seminal vesicle area in pairs (white bars) and octets (grey bars) for the three measurement days (Day 0, 2 and 4). Error bars represent standard errors. (b) Relationships between testis area and the increase in seminal vesicle area from day 0 to day 4 ( $\triangle$ seminal vesicle area) for pairs (white squares) and octets (grey squares). The lines represent linear regressions for pairs (dashed) and octets (solid) (see Results for statistics) 
octets (ANCOVA, $r^{2}=0.44$, factor: group size, $F_{1,46}=7.87, P=0.007$, covariate: body size, $\left.F_{1,46}=25.8, P<0.001\right)$.

We found no overall group size effect on seminal vesicle size (univariate repeated measures ANOVA, between subjects: effect of group size, $F_{1,47}=0.2, P=0.66$, Fig. 3a). Seminal vesicle size increased very strongly with from "Day 0" to "Day 4" in both treatment groups (within subjects: effect of time, $F_{2,94}=328.2, P<0.001$, Fig. 3a). Moreover, the highly significant interaction term between group size and time indicates that the worms in octets not only started with a lower seminal vesicle size on "Day 0", but also ended with a higher seminal vesicle size on "Day 4" than worms from pairs (interaction between group size and time, $F_{2,94}=14.8, P<0.001$, Fig. 3a), suggesting that worms in octets clearly have a larger sperm production rate.

Although testis size explained the largest part of the increase in seminal vesicle size from "Day 0" to "Day 4", there was a significant effect of the treatment group that was independent of testis size (ANCOVA, $r^{2}=0.56$, factor: group size, $F_{1,46}=9.76, P=0.003$, covariate: testis size, $F_{1,46}=30.2, P<0.001$, Fig. $3 \mathrm{~b}$ ), suggesting that testis size is an incomplete measure of sperm production rate.

\section{Discussion}

We have shown here that, in the free-living flatworm Macrostomum lignano, the previously identified phenotypically plastic adjustment of male allocation in response to being raised in a group is not only manifest as an increase in testis size (Schärer and Ladurner 2003; Schärer et al. 2005; Brauer et al. in press) and testicular cell proliferation activity (Schärer et al. 2004b), but also as an increase in sperm production rate. In addition, we have identified a component in sperm production rate, which is independent of variation in testis size: worms grown in larger groups had a higher sperm production rate per unit testicular tissue than worms grown in pairs (i.e. they had a higher spermatogenic efficiency). This suggests that, although testis size appears to be an important predictor of sperm production rate, we have identified a novel component that is ignored by just assuming a simple relationship between testis size and sperm production rate. In the following sections we discuss possible causes for this testis-size-independent component, and suggest future studies to test them.

Adjustment of the speed of spermatogenesis

An interesting possibility to explain this results is that worms modulate the speed of spermatogenesis in response to group size. There is some published information on variation in spermatogenesis speed between different strains of mice and rats (Amann 1970), but these differences presumably have a genetic basis. We are not aware of studies that have shown that such variation can be phenotypically plastic. Nevertheless we consider this a likely explanation for the observed effect.

As outlined in the introduction, studies in birds and mammals often use the number of homogenization resistant spermatid nuclei to estimate the number of sperm currently in production. They then divide this number by the amount of time it takes from this stage to the finished sperm (i.e. called time divisor, for details see Amann 1970), which is of course a function of the speed of spermatogenesis. Usually the time divisor is measured using DNA labelling with either ${ }^{3} \mathrm{H}$-thymidine or 
halogenated pyrimidines (e.g. bromodeoxyuridine, BrdU). It is generally treated as a species-specific value (Amann 1970), but sometimes even used across fairly unrelated species (e.g. Tuttle and Pruett-Jones 2004 use a measure for Japanese quail to make inferences about sperm production rates in fairy wrens). However, as Amann (1970) states: 'Variation, among and within individuals, in the duration of one cycle of the seminiferous epithelium is small but represents an important source of error when calculating sperm production'.

Our data suggest that this variation may be considerable, and that it could occur as a phenotypically plastic response to different levels of sperm competition. Spermatogenesis speed may thus be a more variable trait than previously thought. We have recently established a method that allows BrdU-labelling of sperm nuclei in $M$. lignano (Schärer et al. in press), so we can in the future directly test for timing differences in spermatogenesis between worms raised in pairs and octets.

\section{Phenotypic plasticity in sperm size}

Another interesting possibility would be that exposing worms to different group sizes leads to changes in sperm size. In order to explain our results, worms in groups would have to produce larger sperm, which would lead to a faster increase in seminal vesicle size with the same number of sperm. Larger sperm could be advantageous in the context of sperm competition. The sperm of $M$. lignano are highly complex structures, and appear to compete with each other for access to the cellular valve, which is a specialized tissue in the female sperm-receiving organ, the female antrum (see Schärer et al. 2004a; Ladurner et al. 2005 for more details on sperm anatomy).

We are aware of only one published study that explicitly reports phenotypic plasticity in sperm size, which found complex responses in sperm size to changes in temperature (Hellriegel and Blanckenhorn 2002). However, the relationship of this variation to sperm competition was unclear. Nevertheless, sperm size is a very variable trait that appears to correlate with variation in sperm competition, both within (e.g. Radwan 1996; LaMunyon and Ward 1998, 2002), and between species (e.g. Gage 1994; LaMunyon and Ward 1999; Morrow and Gage 2000, but see Hosken 1997; Gage and Freckleton 2003). We thus see no a priori reason why phenotypically plastic sperm size should not occur, and suggest that such effects should be tested.

\section{Relationship between sperm number and seminal vesicle size}

Finally, we need to evaluate the possibility that the observed effect is an artefact. This could occur if the slope of the relationship between sperm number and seminal vesicle size were steeper outside the range covered by the pairs (which have a smaller range in seminal vesicle size, see Fig. 3a). If so, we would underestimate the sperm production rate of the pairs. This would, for example, occur if in small seminal vesicles relatively few additional sperm would lead to a relatively large increase in seminal vesicle size. We could then overestimate the sperm production of worms in groups, as they on average started with smaller seminal vesicles (Fig. 3a). However, although we tested a number of non-linear functions to describe the relationship between seminal vesicle size and sperm number, none gave a better fit to the data than the most parsimonious linear fit (Fig. 2). We therefore do not think that the observed difference in the testis-size-independent component in sperm production between worms raised in pairs and groups can be explained by mere changes in the 
arrangement of stored sperm, but that it instead represents an actual difference in sperm production rate per unit testis.

\section{Conclusions}

We have previously shown that $M$. lignano responds to increased group size by increasing its testis size. Here we show that increased group size also leads to a higher sperm production rate. However, sperm production rate increased more than expected by the increase in testis size alone, which may suggest that worms raised in groups have a faster spermatogenesis and/or produce larger sperm. In future studies we aim at evaluating these possibilities.

We conclude that, rather than just assuming the relationship between testis size and sperm production rate, this relationship should actually be tested experimentally. Moreover, given the evidence for variation in spermatogenic efficiency that we mention in the introduction, and the considerable variation in our within-species comparison, a better understanding of the relationship between testis size and sperm production rate is important for cross-species comparisons of male reproductive investment. Finally, it appears that sperm competition can have previously unknown effects on sperm production, and that the evolution of male allocation in response to sperm competition affects traits other than testis size.

Acknowledgements We would like to thank Christof Seifarth, Peter Ladurner and Johanna Zaubzer for help and support in the laboratory. Giorgina Bernasconi, Bernhard Egger, Dominique Joly, Peter Ladurner and Klaus Reinhardt provided helpful discussion and comments on the manuscript. During this study L.S. was supported by a Lise-Meitner-fellowship (FWF, Austria), an advanced researcher fellowship (SNF, Switzerland), and a start-up fellowship of the Tyrolean Science Foundation (TWF, Austria).

\section{References}

Amann RP (1970) Sperm production rates. In: Johnson AD, Gomes WR, Vandermark NL (eds) The testis. Academic Press, New York, pp 433-482

Andersen RA, Berges JA, Harrison PJ, Watanabe MM (2005) Recipes for freshwater and seawater media. In: Andersen RA (ed) Algal culturing techniques. Elsevier, Amsterdam, pp 429-538

Aspbury AS, Gabor CR (2004a) Differential sperm priming by male sailfin mollies (Poecilia latipinna): effects of female and male size. Ethology 110:193-202

Aspbury AS, Gabor CR (2004b) Discriminating males alter sperm production between species. Proc Natl Acad Sci USA 101:15970-15973

Bozynski CC, Liley NR (2003) The effect of female presence on spermiation, and of male sexual activity on 'ready' sperm in the male guppy. Anim Behav 65:53-58

Brauer VS, Schärer L, Michiels NK (in press) Phenotypically flexible sex allocation in a simultaneous hermaphrodite. Evolution

Byrne PG, Roberts JD, Simmons LW (2002) Sperm competition selects for increased testes mass in Australian frogs. J Evol Biol 15:347-355

Cooke PS, Porcelli J, Hess RA (1992) Induction of increased testis growth and sperm production in adult rats by neonatal administration of the goitrogen propylthiouracil (PTU): the critical period. Biol Reprod 46:146-154

Cooke PS, Zhao YD, Hansen LG (1996) Neonatal polychlorinated biphenyl treatment increases adult testis size and sperm production in the rat. Toxicol Appl Pharmacol 136:112-117

de Reviers M. Williams JB (eds) (1984) Testis development and production of spermatozoa in the cockerel (Gallus domesticus). British Poultry Science, Harlow

A) Springer 
Evans JP, Magurran AE (1999) Geographic variation in sperm production by Trinidadian guppies. Proc R Soc Lond B 266:2083-2087

Gage MJG (1994) Associations between body size, mating pattern, testis size and sperm lengths across butterflies. Proc R Soc Lond B 258:247-254

Gage MJG, Freckleton RP (2003) Relative testis size and sperm morphometry across mammals: no evidence for an association between sperm competition and sperm length. Proc R Soc Lond B 270:625-632

Gage MJG, Stockley P, Parker GA (1995) Effects of alternative male strategies on characteristics of sperm production in the Atlantic salmon (Salmo salar): theoretical and empirical investigations. Phil Trans R Soc Lond B 350:391-399

Gupta G, Maikhuri JP, Dwivedi AK, Dhar JD, Setty BS (1999) Changes in daily sperm production rate in rats under the influence of a potent antispermatogenic agent, CDRI 84/35. Contraception 59:401-404

Hellriegel B, Blanckenhorn WU (2002) Environmental influences on the gametic investment of yellow dung fly males. Evol Ecol 16:505-522

Hosken DJ (1997) Sperm competition in bats. Proc R Soc Lond B 264:385-392

Johnson RK, Eckardt GR, Rathje TA, Drudik DK (1994) 10 generations of selection for predicted weight of testes in swine - direct response and correlated response in body-weight, backfat, age at puberty, and ovulation rate. J Anim Sci 72:1978-1988

Ladurner P, Rieger RM, Baguña J (2000) Spatial distribution and differentiation potential of stem cells in hatchlings and adults in the marine platyhelminth Macrostomum sp.: a bromodeoxyuridine analysis. Dev Biol 226:231-241

Ladurner P, Schärer L, Salvenmoser W, Rieger RM (2005) A new model organism among the lower Bilateria and the use of digital microscopy in taxonomy of meiobenthic Platyhelminthes: Macrostomum lignano, n. sp (Rhabditophora, Macrostomorpha) J Zool Syst Evol Res 43:114-126

LaMunyon CW, Ward S (1998) Larger sperm outcompete smaller sperm in the nematode Caenorhabditis elegans. Proc R Soc Lond B 265:1997-2002

LaMunyon CW, Ward S (1999) Evolution of sperm size in nematodes: sperm competition favours larger sperm. Proc R Soc Lond B 266:263-267

LaMunyon CW, Ward S (2002) Evolution of larger sperm in response to experimentally increased sperm competition in Caenorhabditis elegans. Proc R Soc Lond B 269:1125-1128

Malo AF, Roldan ER S, Garde J, Soler AJ, Gomendio M (2005) Antlers honestly advertise sperm production and quality. Proc R Soc Lond B 272:149-157

Minder AM, Hosken DJ, Ward PI (2005) Co-evolution of male and female reproductive characters across the Scathophagidae (Diptera). J Evol Biol 18:60-69

Møller AP (1989) Ejaculate quality, testes size and sperm production in mammals. Funct Ecol 3:9196

Moreira JR, Macdonald DW, Clarke JR (1997) Correlations of testis mass in capybaras (Hydrochaeris hydrochaeris): dominance assurance of sperm competition. J Zool 241:457-463

Morrow EH, Gage MJG (2000) The evolution of sperm length in moths. Proc R Soc Lond B 267:307-313

Nakatsuru K, Kramer DL (1982) Is sperm cheap? Limited male fertility and female choice in the lemon tetra (Pisces, Characidae). Science 216:753-755

Newlon AW, Yund PO, Stewart-Savage J (2003) Phenotypic plasticity of reproductive effort in a colonial ascidian, Botryllus schlosseri. J Exp Zool A 297A:180-188

Parker GA (1998) Sperm competition and the evolution of ejaculates: towards a theory base. In: Birkhead TR, Møller AP (eds) Sperm competition and sexual selection. Academic Press, London, England, pp 3-54

Peirce EJ, Breed WG (2001) A comparative study of sperm production in two species of Australian arid zone rodents (Pseudomys australis, Notomys alexis) with marked differences in testis size. Reproduction 121:239-247

Pilastro A, Scaggiante M, Rasotto MB (2002) Individual adjustment of sperm expenditure accords with sperm competition theory. Proc Natl Acad Sci USA 99:9913-9915

Pitnick S, Markow TA (1994a) Large-male advantages associated with costs of sperm production in Drosophila hydei, a species with giant sperm. Proc Natl Acad Sci USA 91:9277-9281

Pitnick S, Markow TA (1994b) Male gametic strategies: sperm size, testes size, and the allocation of ejaculate among successive mates by the sperm-limited fly Drosophila pachea and its relatives. Am Nat 143:785-819

Pitnick S, Miller GT, Reagan J, Holland B (2001) Males' evolutionary responses to experimental removal of sexual selection. Proc R Soc Lond B 268:1071-1080 
Radwan J (1996) Intraspecific variation in sperm competition success in the bulb mite: a role for sperm size. Proc R Soc Lond B 263:855-859

Rathje TA, Johnson RK, Lunstra DD (1995) Sperm production in boars after 9 generations of selection for increased weight of testis. J Anim Sci 73:2177-2185

Rieger RM, Gehlen M, Haszprunar G, Holmlund M, Legniti A, Salvenmoser W, Tyler S (1988) Laboratory cultures of marine Macrostomida (Turbellaria). Fortschr Zool 36:523

Sall J, Lehman A (1996) JMP start statistics: A guide to statistical and data analysis using JMP, JMPIN software. Duxbury Press, Belmont, USA

Schärer L, Joss G, Sandner P (2004a) Mating behaviour of the marine turbellarian Macrostomum sp.: these worms suck. Mar Biol 145:373-380

Schärer L, Ladurner P (2003) Phenotypically plastic adjustment of sex allocation in a simultaneous hermaphrodite. Proc R Soc Lond B 270:935-941

Schärer L, Ladurner P, Rieger RM (2004b) Bigger testes do work more: experimental evidence that testis size reflects testicular cell proliferation activity in the marine invertebrate, the free-living flatworm Macrostomum sp. Behav Ecol Sociobiol 56:420-425

Schärer L, Sandner P, Michiels NK (2005) Trade-off between male and female allocation in the simultaneously hermaphroditic flatworm Macrostomum sp. J Evol Biol 18:396-404

Schärer L, Zaubzer J, Salvenmoser W, Seifarth C, Ladurner P (in press) Tracking sperm of a donor in a recipient: an immunocytochemical approach. Animal Biol

Tuttle EM, Pruett-Jones S (2004) Estimates of extreme sperm production: morphological and experimental evidence from reproductively promiscuous fairy-wrens (Malurus). Anim Behav 68:541-550

Tuttle EM, Pruett-Jones S, Webster MS (1996) Cloacal protuberances and extreme sperm production in Australian fairy-wrens. Proc R Soc Lond B 263:1359-1364

Tyler S (1981) Development of cilia in embryos of the turbellarian Macrostomum. Hydrobiologia 84:231-239

Wedell N, Gage MJG, Parker GA (2002) Sperm competition, male prudence and sperm-limited females. Trends Ecol Evol 17:313-320

Wistuba J, Schrod A, Greve B, Hodges JK, Aslam H, Weinbauer GF, Luetjens CM (2003) Organization of seminiferous epithelium in primates: relationship to spermatogenic efficiency, phylogeny, and mating system. Biol Reprod 69:582-591 